\title{
Neutrino spin rotation in dense matter and electromagnetic field
}

\author{
E. V. Arbuzova, ${ }^{1}$ A. E. Lobanov, ${ }^{2}$ and E. M. Murchikova ${ }^{3}$ \\ 1 International University "Dubna", 141980 Dubna, Russia \\ E-mail: arbuzova@uni-dubna.ru \\ 2 Department of Theoretical Physics, Moscow State University, 119991 Moscow, \\ Russia \\ E-mail: lobanov@phys.msu.ru \\ 3 Department of Theoretical Physics, Moscow State University, 119991 Moscow, \\ Russia \\ E-mail: murchikova@nanolab.phys.msu.ru \\ PACS numbers: 03. 50. De, 12. 20. Ds
}

\begin{abstract}
Exact solutions of the Dirac-Pauli equation for massive neutrino with anomalous magnetic moment interacting with dense matter and strong electromagnetic field are found. The complete system of neutrino wavefunctions, which show spin rotation properties are obtained and their possible applications are discussed.
\end{abstract}

\section{Introduction}

The neutrino oscillation phenomenon is well established now [1]. For the detailed information about neutrino flavour oscillations we refer to the pioneer works [2, 3], some resent reviews [4, 5] and references therein. From the theory of this phenomenon it follows that oscillations are possible only when neutrino possesses non-vanishing mass. Consequently, a neutrino has non-trivial electromagnetic properties. In particular, a Dirac neutrino has to possess a non-zero magnetic moment [6]. In turn, the existence of the magnetic moment is associated with so-called neutrino spin oscillations. These oscillations are described as neutrino spin precession or, in other words, as transitions between left-handed (active) and right-handed (sterile) neutrino states in an electromagnetic field. Phenomenology of this effect was widely discussed in the literature [7, 8, 9, 10, 11, 12, 13, 14, 15, 16].

However in the most popular papers the theory of neutrino spin oscillation was based on solving the Cauchy problem for the Schrödinger type equation with an effective Hamiltonian. So the states of neutrino were treated as mixed states. The purpose of our work is to find a complete system of solutions of the wave equation which accounts for the influence of an electromagnetic field and a dense matter on neutrino dynamics. 
The existence of such system gives a possibility to describe the states of neutrino with rotating spin as pure states and to evaluate the probabilities of various processes with neutrino in the framework of the Furry picture.

\section{Model}

When the interaction of a massive neutrino possessing an anomalous magnetic moment $\mu_{0}$ with background fermions is considered to be coherent, its propagation in matter and electromagnetic field is described by the Dirac-Pauli equation with the effective potential [17, 18]. For a moving and polarized matter such an interaction is described by the effective four-potential $f^{\mu}$.

The function

$$
f^{\mu}=\sum_{f} \rho_{f}^{(1)} j_{f}^{\mu}+\rho_{f}^{(2)} \lambda_{f}^{\mu}
$$

is a linear combination of the currents

$$
j_{f}^{\mu}=\left\{n_{f} u_{f}^{0}, n_{f} \mathbf{u}_{f}\right\},
$$

and the polarizations

$$
\lambda_{f}^{\mu}=\left\{n_{f}\left(\boldsymbol{\zeta}_{f} \mathbf{u}_{f}\right), n_{f}\left(\boldsymbol{\zeta}_{f}+\frac{\mathbf{u}_{f}\left(\boldsymbol{\zeta}_{f} \mathbf{u}_{f}\right)}{1+u^{0}}\right)\right\}
$$

of background fermions. In these equations $n_{f}$ and $\boldsymbol{\zeta}_{f}\left(0 \leqslant\left|\boldsymbol{\zeta}_{f}\right|^{2} \leqslant 1\right)$ are, respectively, the number density and the mean value of the polarization vector of the background fermions $f$ in their center-of-mass system. In this reference frame the mean momentum of the fermions $f$ is equal to zero, and $u_{f}^{\mu}=\left\{u_{f}^{0}, \mathbf{u}_{f}\right\}$ denote the four-velocity of the reference frame. Summation is carried out over the background fermions $f$. We use the units $\hbar=c=1$.

The expressions for the coefficients $\rho_{f}^{(1,2)}$ depend on the model chosen for neutrino interactions. In the framework of the standard model these coefficients calculated in the first order of the perturbation theory have the forms [19, 20, 21, 22]

$$
\rho_{f}^{(1)}=\sqrt{2} G_{\mathrm{F}}\left\{I_{e \nu}+T_{3}^{(f)}-2 Q^{(f)} \sin ^{2} \theta_{\mathrm{W}}\right\}, \quad \rho_{f}^{(2)}=\sqrt{2} G_{\mathrm{F}}\left\{I_{e \nu}+T_{3}^{(f)}\right\} .
$$

Here $Q^{(f)}$ is an electric charge of the fermion, $T_{3}^{(f)}$ is a third component of weak isospin, $G_{\mathrm{F}}$ and $\theta_{\mathrm{W}}$ are the Fermi constant and the Weinberg angle respectively. For electron neutrino interaction with electrons $I_{e \nu}=1$, positrons $I_{e \nu}=-1$, otherwise $I_{e \nu}=0$.

Let us discuss an equation for neutrino (its mass eigenstate) in matter and electromagnetic field. In order to avoid correlations between flavour and spin oscillations (the Mikheyev-Smirnov-Wolfenstein effect [17, 18]) we shall suppose that the effective four-potential is the same for all neutrino flavours and consider the matter, in which a fraction of electrons is small, i.e. approximately $n_{e}=0$. In what follows, we restrict our consideration to the case of a homogeneous medium. Then the explicit form of the 
generalized Dirac-Pauli equation is uniquely determined by the assumptions similar to those adopted in [23]:

$$
\left(i \hat{\partial}-\frac{1}{2} \hat{f}\left(1+\gamma^{5}\right)-\frac{i}{2} \mu_{0} F^{\mu \nu} \sigma_{\mu \nu}-m\right) \Psi=0
$$

Here $F^{\mu \nu}$ is an electromagnetic field tensor, $\sigma_{\mu \nu}=\frac{1}{2}\left(\gamma^{\mu} \gamma^{\nu}-\gamma^{\nu} \gamma^{\mu}\right)$. The quantities with hats denote scalar products of the Dirac matrices with four-vectors: $\hat{a} \equiv \gamma^{\mu} a_{\mu}$.

Note, when propagation of neutrino through a dense matter and an electromagnetic field is studied, we should keep in mind that the matter and the field are mainly situated in the same area. Therefore strengthes of electric and magnetic fields and average velocities and polarizations of matter should satisfy the self-consistent system of equations including the Maxwell equations, the Lorentz equation

$$
\dot{u}_{f}^{\mu}=\frac{e_{f}}{m_{f}} F_{\nu}^{\mu} u_{f}^{\nu},
$$

and the classical spin evolution Bargmann-Michel-Telegdi (BMT) equation [24]

$$
\dot{\lambda}^{\mu}=\left[\frac{e_{f}}{m_{f}} F_{\nu}^{\mu}+2 \mu_{f}\left(F_{\nu}^{\mu}-u_{f}^{\mu} F_{\alpha \nu} u_{f}^{\alpha}\right)\right] \lambda_{f}^{\nu} .
$$

Here a dot denotes the differentiation with respect to the proper time $\tau$.

As already mentioned, we restrict our consideration to constant velocity and polarization of matter and constant homogeneous electromagnetic field in equation (5). However, even such a choice imposes certain limitations on $F^{\mu \nu}$ and $f^{\mu}$. Indeed, function $f^{\mu}$ is a linear combination of currents and polarizations of background fermions. Since the velocities, polarizations and number densities of different components of matter are independent characteristics in the general case, then from the condition $f^{\mu}=$ const or $\dot{f}_{\mu}=0$ it follows that velocities and polarizations of background components should be stationary: $\dot{u}_{f}^{\mu}=0, \dot{\lambda}_{f}^{\mu}=0$. Using equations (6), (7) we can obtain the restriction

$$
F^{\mu \nu} f_{\nu}=0 \text {. }
$$

It should be stressed that this condition is a direct consequence of the fact that the average velocity and polarization of particles of matter in an external field should satisfy the classical equations of motion.

\section{Calculations}

Let us look for a solution of equation (5). Since the functions $F^{\mu \nu}, f^{\mu}$ are constant, the canonical momentum operator $i \partial_{\mu}$ commutes with the Hamiltonian of this equation. However, the commonly adopted choice of eigenvalues of this operator as quantum numbers is not satisfactory in our case. Particle kinetic momentum components related to its group four-velocity $u^{\mu}$ by the relation $q^{\mu}=m u^{\mu}, q^{2}=m^{2}$ are more suitable to play this role. In the present paper we discuss the solutions described by the quantum numbers which can be interpreted as kinetic momentum components. The explicit form of a kinetic momentum operator for a particle with spin is not known beforehand, hence, in order to find the appropriate solutions, we have to use the correspondence principle. 
It was shown in [25] that if effects of neutrino weak interactions are taken into account, the Lorentz invariant generalization of the BMT equation for a spin vector $S^{\mu}$ of a neutrino moving with the four-velocity $u^{\mu}$ has the form

$$
\dot{S}^{\mu}=2\left\{\left(F^{\mu \nu}+G^{\mu \nu}\right) S_{\nu}-u^{\mu} u_{\nu}\left(F^{\nu \lambda}+G^{\nu \lambda}\right) S_{\lambda}\right\},
$$

where

$$
G^{\mu \nu}=\frac{1}{2} e^{\mu \nu \rho \lambda} f_{\rho} u_{\lambda}
$$

Here and further for the simplification of the formulae we include a value of neutrino magnetic moment into an electromagnetic field tensor, i.e. $\mu_{0} F^{\mu \nu} \Rightarrow F^{\mu \nu}$.

We introduce quasi-classical spin wavefunctions, which can be constructed as discussed in [26, 27]. Suppose the Lorentz equation is solved, and the dependence of particle coordinates on the proper time is found. Then the BMT equation transforms to an ordinary differential equation, whose resolvent determines a one-parametric subgroup of the Lorentz group. The quasi-classical spin wavefunction is represented by a spintensor, whose evolution is determined by the same one-parametric subgroup. In our case this spin-tensor represents the Dirac bispinor.

Following the technique presented in the works [26, 28] we choose the solution of equation (5) in the form

$$
\Psi(x)=e^{-i F(x)} U\left(\tau(x), \tau_{0}=0\right) \Psi_{0}(x) .
$$

In this formula $U\left(\tau, \tau_{0}\right)$ is an operator of evolution for a quasi-classical spin wavefunction, $e^{-i F(x)}$ is a phase factor, and

$$
\Psi_{0}(x)=e^{-i q x}\left(1-\zeta_{0} \gamma^{5} \hat{S}_{0}\right)(\hat{q}+m) \psi_{0}
$$

is a solution of the Dirac equation for a free particle. Here four-vector $S_{0}^{\mu}$ determines the direction of particle polarization, $\zeta_{0}= \pm 1$ is a sign of spin projection on this direction and $\psi_{0}$ is a constant bispinor normalized by the condition $\bar{\Psi}_{0}(x) \Psi_{0}(x)=m / q_{0}$.

In our case operator $U\left(\tau, \tau_{0}\right)$ obeys the equation

$$
\dot{U}\left(\tau, \tau_{0}\right)=\left\{\frac{i}{4 m} \gamma^{5}(\hat{f} \hat{q}-\hat{q} \hat{f})+\frac{i}{m^{2}} \gamma^{5} H^{\mu \nu} q_{\nu} \gamma_{\mu} \hat{q}\right\} U\left(\tau, \tau_{0}\right),
$$

where $H^{\mu \nu}=-\frac{1}{2} e^{\mu \nu \rho \lambda} F_{\rho \lambda}$ is a dual electromagnetic field tensor. It is obvious that the solution of equation (131) can be represented as a matrix exponent

$$
U\left(\tau, \tau_{0}\right)=\exp \left\{i\left(\tau-\tau_{0}\right)\left[\frac{1}{4 m} \gamma^{5}(\hat{f} \hat{q}-\hat{q} \hat{f})+\frac{1}{m^{2}} \gamma^{5} H^{\mu \nu} q_{\nu} \gamma_{\mu} \hat{q}\right]\right\} .
$$

Substitution of the expression (11) into equation (5) taking into account (13) leads to the relation

$$
\begin{aligned}
& \left\{\hat{q}+\hat{\partial} F(x)-\frac{1}{2} \hat{f}+\frac{1}{2} \gamma^{5} \hat{f}+\gamma^{5} \hat{N}\left[\frac{1}{4 m}(\hat{f} \hat{q}-\hat{q} \hat{f})+\frac{1}{m^{2}} H^{\mu \nu} q_{\nu} \gamma_{\mu} \hat{q}\right]\right. \\
& \left.-\frac{i}{2} F^{\mu \nu} \sigma_{\mu \nu}-m\right\} e^{-i F(x)} U\left(\tau(x), \tau_{0}\right) \Psi_{0}(x)=0
\end{aligned}
$$


where $N^{\mu}=\partial^{\mu} \tau$. Since the commutator $[\hat{q}, U]=0$, and the matrix $U\left(\tau(x), \tau_{0}\right)$ is nondegenerate, then to hold this relation the following condition is required:

$$
\begin{aligned}
& \hat{\partial} F(x)-\frac{1}{2} \hat{f}+\frac{1}{2} \gamma^{5} \hat{f}+\gamma^{5} \hat{N}\left[\frac{1}{4 m}(\hat{f} \hat{q}-\hat{q} \hat{f})+\frac{1}{m^{2}} H^{\mu \nu} q_{\nu} \gamma_{\mu} \hat{q}\right] \\
& -\frac{i}{2} F^{\mu \nu} \sigma_{\mu \nu}=0 .
\end{aligned}
$$

Since the coefficients at linearly independent elements of the Dirac matrix algebra must be equal to zero independently, we receive

$$
\partial^{\mu} F(x)=f^{\mu} / 2,
$$

and the system of equations for determination of the vector $N^{\mu}$ :

$$
\varphi^{\mu}(m-(N q))+(N \varphi) q^{\mu}=0, \quad F^{\mu \alpha} q_{\alpha}=-e^{\mu \nu \rho \lambda} N_{\nu} \varphi_{\rho} q_{\lambda},
$$

where $\varphi^{\mu}=f^{\mu} / 2+H^{\mu \nu} q_{\nu} / m$.

It is easily verified that for the compatibility of this system it is necessary

$$
q_{\mu} F^{\mu \nu} H_{\nu \alpha} q^{\alpha}+m q_{\mu} F^{\mu \nu} f_{\nu} / 2=0 .
$$

Since $q^{\mu}$ takes in general an arbitrary value, in order to satisfy (17) identically it is necessary to demand the conditions

$$
\begin{aligned}
& F^{\mu \alpha} H_{\alpha \nu} \equiv-\frac{1}{4} \delta_{\nu}^{\mu} F^{\alpha \beta} H_{\alpha \beta}=0, \\
& F^{\mu \nu} f_{\nu}=0 .
\end{aligned}
$$

Hence, the solution of equation (5) can take the form (11) only if the tensor $F^{\mu \nu}$ is flat, i.e. its second invariant $I_{2}=\frac{1}{4} F^{\mu \nu} H_{\mu \nu}$ is equal to zero, and the vector $f^{\mu}$ is its eigenvector corresponding to the zero eigenvalue. It should be noted that the antisymmetric tensor has an eigenvector corresponding to zero eigenvalue if and only if this tensor is flat. So conditions (18) and (19) are not independent, and (18) follows directly from (19). Thus we come to the condition above (8), which was obtained only on the physical attends.

Let us introduce an orthogonal basis in the Minkowski space

$$
n_{0}^{\mu}=q^{\mu} / m, \quad n_{1}^{\mu}=\frac{H^{\mu \nu} q_{\nu}}{\sqrt{\mathcal{N}}}, \quad n_{2}^{\mu}=\frac{F^{\mu \nu} q_{\nu}}{\sqrt{\tilde{\mathcal{N}}}}, \quad n_{3}^{\mu}=\frac{m^{2} H^{\mu \nu} H_{\nu \alpha} q^{\alpha}-q^{\mu} \mathcal{N}}{m \sqrt{\mathcal{N} \tilde{\mathcal{N}}}},
$$

where $\mathcal{N}=q_{\mu} H^{\mu \nu} H_{\nu \rho} q^{\rho}, \tilde{\mathcal{N}}=q_{\mu} F^{\mu \nu} F_{\nu \rho} q^{\rho}$. Expanding the four-vector $N^{\mu}$ with the basis and using equations (16) we get as the result of calculations

$$
N^{\mu}=n_{0}^{\mu}+n_{1}^{\mu} \frac{(\varphi q)\left[\mathcal{N}-\tilde{\mathcal{N}}-m f_{\mu} H^{\mu \nu} q_{\nu}\right]}{2 \sqrt{\mathcal{N}}\left((\varphi q)^{2}-m^{2} \varphi^{2}\right)}+n_{3}^{\mu} \frac{\sqrt{\tilde{\mathcal{N}}}}{\sqrt{\mathcal{N}}}\left[1+\frac{m^{2}(f \varphi)}{2\left((\varphi q)^{2}-m^{2} \varphi^{2}\right)}\right] .
$$

With the help of the relations

$$
\begin{aligned}
& 2(\varphi q) H^{\mu \nu}=m\left(\varphi^{\mu} f^{\nu}-f^{\mu} \varphi^{\nu}\right), \quad \mathcal{N}-\tilde{\mathcal{N}}=2 m^{2} I_{1} \\
& \left((f \varphi)^{2}-f^{2} \varphi^{2}\right) m^{2}=\left(f^{2} \mathcal{N}+\left(f_{\mu} H^{\mu \nu} q_{\nu}\right)^{2}\right)=8(\varphi q)^{2} I_{1}
\end{aligned}
$$


where $I_{1}=\frac{1}{4} F^{\mu \nu} F_{\mu \nu}$ is the first invariant of the tensor $F^{\mu \nu}$, expression (20) can be represented as

$$
N^{\mu}=-q^{\mu} \frac{m(f \varphi)}{2\left((\varphi q)^{2}-m^{2} \varphi^{2}\right)}+f^{\mu} \frac{m}{2(\varphi q)}+\varphi^{\mu} \frac{m^{3}(f \varphi)}{2(\varphi q)\left((\varphi q)^{2}-m^{2} \varphi^{2}\right)} .
$$

Let us note that, in order to derive formulae of the form (21), one should take the following arguments into consideration. For an arbitrary antisymmetric tensor $A^{\mu \nu}$, its dual tensor ${ }^{\star} A^{\mu \nu}=-\frac{1}{2} e^{\mu \nu \rho \lambda} A_{\rho \lambda}$, and any four-vectors $g^{\mu}, h^{\mu},(g h) \neq 0$ the following relation takes place

$$
A^{\mu \nu}(g h)=-\left[g^{\mu} A^{\nu \rho} h_{\rho}-A^{\mu \rho} h_{\rho} g^{\nu}\right]+{ }^{\star}\left[h^{\mu \star} A^{\nu \rho} g_{\rho}-{ }^{\star} A^{\mu \rho} g_{\rho} h^{\nu}\right] .
$$

It leads to

$$
\begin{aligned}
& g_{\mu}{ }^{\star} A_{\rho}^{\mu \star} A_{\nu}^{\rho} g^{\nu} h^{2}+\left(g_{\mu}{ }^{\star} A_{\nu}^{\mu} h^{\nu}\right)^{2}-h_{\mu} A_{\rho}^{\mu} A_{\nu}^{\rho} h^{\nu} g^{2}-\left(g_{\mu} A_{\nu}^{\mu} h^{\nu}\right)^{2}= \\
& =g_{\mu}{ }^{\star} A_{\rho}^{\mu \star} A_{\nu}^{\rho} h^{\nu}(g h)-g_{\mu} A_{\rho}^{\mu} A_{\nu}^{\rho} h^{\nu}(g h)=2(g h)^{2} I_{1} .
\end{aligned}
$$

Formulae (21) follow from equation (24) if conditions (18), (19) are taken into account.

Since $f^{\mu}, N^{\mu}=$ const we obtain for the proper time

$$
\tau=(N x),
$$

and for the phase factor which determines energy shift of neutrino in matter we have

$$
F(x)=(f x) / 2 .
$$

It is not difficult to verify that in view of the relations obtained, the expression for the wavefunction takes the form

$$
\Psi(x)=\frac{1}{2} \sum_{\zeta= \pm 1} e^{-i\left(P_{\zeta} x\right)}\left(1-\zeta \gamma^{5} \hat{S}_{t p}\right)\left(1-\zeta_{0} \gamma^{5} \hat{S}_{0}\right)(\hat{q}+m) \psi_{0}
$$

where

$$
\begin{gathered}
S_{t p}^{\mu}=\frac{q^{\mu}(\varphi q) / m-\varphi^{\mu} m}{\sqrt{(\varphi q)^{2}-\varphi^{2} m^{2}}} \\
P_{\zeta}^{\mu}=q^{\mu}+f^{\mu} / 2-\zeta N^{\mu} \sqrt{(\varphi q)^{2}-\varphi^{2} m^{2}} / m=q^{\mu}\left(1+\zeta \frac{(f \varphi)}{2 \sqrt{(\varphi q)^{2}-m^{2} \varphi^{2}}}\right) \\
+\frac{1}{2} f^{\mu}\left(1-\frac{\zeta \sqrt{(\varphi q)^{2}-m^{2} \varphi^{2}}}{(\varphi q)}\right)-\varphi^{\mu} \frac{\zeta(f \varphi) m^{2}}{2(\varphi q) \sqrt{(\varphi q)^{2}-m^{2} \varphi^{2}}}
\end{gathered}
$$

It is obvious that the system of solutions (27) is a complete system of solutions of equation (5), which is characterized by the kinetic momentum of the particle $q^{\mu}$ and the quantum number $\zeta_{0}= \pm 1$, which can be interpreted as the neutrino spin projection on the direction $S_{0}^{\mu}$ at the moment $\tau=(N x)=0$. In the general case this system is not stationary. The received solutions are stationary only when $S_{0}^{\mu}=S_{t p}^{\mu}$. In this case the wavefunctions are the eigenfunctions of the spin projection operator to the direction $S_{t p}^{\mu}$ with the eigenvalues $\zeta= \pm 1$ and of the canonical momentum operator $i \partial^{\mu}$ with the 
eigenvalues $P_{\zeta}^{\mu}$. The orthonormalized system of the stationary solutions of equation (5) can be written down in the following way:

$$
\Psi_{\zeta}(x)=e^{-i\left(P_{\zeta} x\right)} \sqrt{|J|}\left(1-\zeta \gamma^{5} \hat{S}_{t p}\right)(\hat{q}+m) \psi_{0},
$$

where $J$ is the transition Jacobian between the variables $q^{\mu}$ and $P_{\zeta}^{\mu}$ :

$$
J=\operatorname{det}\left(M_{i j}\right)=\operatorname{det}\left[\frac{\partial P_{\zeta}^{i}}{\partial q^{j}}+\frac{\partial P_{\zeta}^{i}}{\partial q^{0}} \frac{\partial q^{0}}{\partial q^{j}}\right] .
$$

Using formulae (21) it is possible to rewrite the matrix $M_{i j}$ in the form

$$
\begin{aligned}
& M_{i j}=\delta_{i j}\left(1+\zeta \frac{(f \varphi)}{2 \sqrt{(\varphi q)^{2}-m^{2} \varphi^{2}}}\right)+ \\
& +\zeta\left(q_{i}-\varphi_{i} \frac{m^{2}}{(\varphi q)}\right)\left(\varphi_{j}-q_{j} \frac{\varphi^{0}}{q^{0}}\right) \frac{m^{2}(f \varphi)^{2}+f^{2}(\varphi q)^{2}-m^{2} f^{2} \varphi^{2}}{4(\varphi q)\left((\varphi q)^{2}-m^{2} \varphi^{2}\right)^{3 / 2}} .
\end{aligned}
$$

It can be easily shown that for any vectors $\mathbf{g}$ and $\mathbf{h}$ the relation

$$
\operatorname{det}\left(\delta_{i j}+g_{i} h_{j}\right)=1+(\mathbf{g h})
$$

is correct. Hence

$$
J=\left(1+\zeta \frac{(f \varphi)}{2 \sqrt{(\varphi q)^{2}-m^{2} \varphi^{2}}}\right)^{2}\left(1+\zeta \frac{f_{\mu} H^{\mu \nu} q_{\nu} / 2 m-2 I_{1}}{\sqrt{(\varphi q)^{2}-m^{2} \varphi^{2}}}\right) .
$$

The structure of solution (30) directly leads us to the conclusion that when neutrinos move through a dense matter and an electromagnetic field which satisfy condition (19), they can behave as free particles, i.e. move with the constant group velocity

$$
\mathbf{v}_{g r}=\frac{\partial P_{\zeta}^{0}}{\partial \mathbf{P}_{\zeta}}=\frac{\mathbf{q}}{q^{0}}
$$

and conserve the polarization.

However in interactions with other particles the channels of reactions which are closed for a free neutrino can be opened (see, for example, [28, 29]), as a result of difference of the dispersion law for the free neutrino $P^{2}=m^{2}$ and the one for neutrino in matter and electromagnetic field:

$$
\tilde{P}^{2}=m^{2}-f^{2} / 4-2 I_{1}-2 \zeta \Delta \sqrt{(\tilde{P} \tilde{\Phi})^{2}-\tilde{\Phi}^{2} m^{2}},
$$

where

$$
\begin{gathered}
\tilde{P}^{\mu}=P_{\zeta}^{\mu}-f^{\mu} / 2, \quad \tilde{\Phi}^{\mu}=f^{\mu} / 2+H^{\mu \nu} \tilde{P}_{\nu} / m, \\
\Delta=\operatorname{sign}\left(1+\zeta \frac{f_{\mu} H^{\mu \nu} q_{\nu} / 2 m-2 I_{1}}{\sqrt{(\varphi q)^{2}-m^{2} \varphi^{2}}}\right) .
\end{gathered}
$$

In order to get equation (33) we used the following relations obtained from formulae (23) and (24)

$$
\begin{gathered}
(\tilde{P} f)=2(\varphi q)\left(1+\zeta \frac{f_{\mu} H^{\mu \nu} q_{\nu} / 2 m-2 I_{1}}{\sqrt{(\varphi q)^{2}-m^{2} \varphi^{2}}}\right), \\
(\tilde{P} f)(f \varphi)=2(\varphi q)(f \tilde{\Phi}), \quad \tilde{\Phi}^{2}(\varphi q)^{2}=\varphi^{2}(\tilde{\Phi} \tilde{P})^{2} .
\end{gathered}
$$


The appearance of the factor $\Delta$ in equation (33) is connected with the fact that $\zeta$ is a particle spin projection on the direction determined by a kinetic particle momentum instead of the canonical one.

Note that the solutions which are classified by a kinetic momentum have been earlier found for equation (5) in which only $F^{\mu \nu} \neq 0$ or only $f^{\mu} \neq 0$ in papers [26] and 28] respectively. These solutions can be received from formula (27) (the normalized stationary solutions from formula ( $(\underline{30})$ ), if either $f^{\mu}=0$ or $F^{\mu \nu}=0$.

\section{Discussion}

Let us discuss the physical meaning of the results obtained. For this purpose we shall consider vector and axial currents constructed with help of solution (27). The vector current is

$$
V^{\mu}=\bar{\Psi}(x) \gamma^{\mu} \Psi(x)=q^{\mu} / q^{0},
$$

i.e. solution (27) describes neutrino propagating with the velocity $\mathbf{v}=\mathbf{q} / q^{0}$. At the same time the axial current is

$$
A^{\mu}=\bar{\Psi}(x) \gamma^{5} \gamma^{\mu} \Psi(x)=\zeta_{0} \frac{m}{q^{0}} S^{\mu}
$$

Here

$$
S^{\mu}=-S_{t p}^{\mu}\left(S_{0} S_{t p}\right)+\left[S_{0}^{\mu}+S_{t p}^{\mu}\left(S_{0} S_{t p}\right)\right] \cos 2 \theta-\frac{1}{m} e^{\mu \nu \rho \lambda} q_{\nu} S_{0 \rho} S_{t p \lambda} \sin 2 \theta,(37
$$

where

$$
\theta=(N x) \sqrt{(\varphi q)^{2}-\varphi^{2} m^{2}} / m .
$$

The spin vector $\boldsymbol{\zeta}$ can be expressed in terms of the four-vector $S^{\mu}$ components as

$$
\boldsymbol{\zeta}=\mathbf{S}-\frac{\mathbf{q} S^{0}}{q^{0}+m}
$$

As the consequence of this fact we have

$$
\boldsymbol{\zeta}=\boldsymbol{\zeta}_{t p}\left(\boldsymbol{\zeta}_{0} \boldsymbol{\zeta}_{t p}\right)+\left[\boldsymbol{\zeta}_{0}-\boldsymbol{\zeta}_{t p}\left(\boldsymbol{\zeta}_{0} \boldsymbol{\zeta}_{t p}\right)\right] \cos 2 \theta-\left[\boldsymbol{\zeta}_{t p} \times \boldsymbol{\zeta}_{0}\right] \sin 2 \theta
$$

Introduce a flight length $L$ of a particle and an oscillations length $L_{\text {osc }}$, using the relation $\theta=\pi L / L_{\text {osc }}$. Since the scalar product $(N x)=\tau$ can be interpreted as the proper time of a particle, then the oscillation length is defined as

$$
L_{o s c}=\frac{2 \pi \hbar c|\mathbf{q}|}{\sqrt{(f q)^{2}-f^{2} m^{2} c^{2}-4 m c \mu_{0} H^{\mu \nu} b_{\mu} q_{\nu}+4 \mu_{0}^{2} H^{\mu \alpha} H_{\alpha \nu} q_{\mu} q^{\nu}}} .
$$

In this formula we use gaussian units and restore the neutrino magnetic moment $\mu_{0}$.

Hence if as a result of a certain process a neutrino arose with polarization $\boldsymbol{\zeta}_{0}$, after

travelling the distance $L$ the probability for the neutrino to have polarization $-\boldsymbol{\zeta}_{0}$ is equal to

$$
W_{s f}=\left[\boldsymbol{\zeta}_{0} \times \boldsymbol{\zeta}_{t p}\right]^{2} \sin ^{2}\left(\pi L / L_{o s c}\right)
$$


Consequently, if the condition $\left(\boldsymbol{\zeta}_{0} \boldsymbol{\zeta}_{t p}\right)=0$ is fulfilled, this probability can become unity, i.e. a resonance takes place.

Thus, solution (27) which is a linear combination of solutions (30) describes a spincoherent state of neutrino, in which a spin rotation takes place. Therefore, neutrino state with rotating spin is a pure state. Existence of such solutions is the direct consequence of the neutrino state description in terms of kinetic momentum. It should be stressed that as the result of calculations we obtained the complete system of neutrino wavefunctions, which show spin rotation properties.

Such a spin behaviour has a simple quasi-classical interpretation. The antisymmetric tensor $G^{\mu \nu}$ (see equation (10) ) can be written in the standard form

$$
G^{\mu \nu}=(\mathbf{P}, \mathbf{M})
$$

where

$$
\mathbf{M}=\left(f^{0} \mathbf{q}-q^{0} \mathbf{f}\right) /(2 m), \quad \mathbf{P}=-[\mathbf{q} \times \mathbf{f}] /(2 m) .
$$

Vectors $\mathbf{P}$ and $\mathbf{M}$ are analogous to the polarization and the magnetization vectors of matter. It should be noted that the substitution $F^{\mu \nu} \Rightarrow F^{\mu \nu}+G_{\mu \nu}$ implies that the magnetic $\mathbf{H}$ and electric $\mathbf{D}$ fields are shifted by the vectors $\mathbf{M}$ and $\mathbf{P}$, respectively:

$$
\mathbf{H} \Rightarrow \mathbf{B}=\mathbf{H}+\mathbf{M}, \quad \mathbf{D} \Rightarrow \mathbf{E}=\mathbf{D}-\mathbf{P} .
$$

So restriction (8) in the explicit form is

$$
(\mathbf{E f})=0, \quad \mathbf{E} f^{0}-[\mathbf{B} \times \mathbf{f}]=0 .
$$

In the rest frame of the particle, equation (9) can be written down in the form

$$
\dot{\boldsymbol{\zeta}}=2\left[\boldsymbol{\zeta} \times \mathbf{B}_{0}\right],
$$

where the spin vector $\boldsymbol{\zeta}$ is related to four-vector $S^{\mu}$ by equation (39) and the value $\mathbf{B}_{0}$ is the effective magnetic field in the neutrino rest frame which can be expressed in terms of quantities determined in the laboratory frame

$$
\begin{aligned}
& \mathbf{B}_{0}=\frac{1}{m}\left[q^{0} \mathbf{B}-[\mathbf{q} \times \mathbf{E}]-\frac{\mathbf{q}(\mathbf{q} \mathbf{B})}{q^{0}+m}\right] \\
& =\frac{1}{m}\left[q^{0} \mathbf{H}-[\mathbf{q} \times \mathbf{D}]-\frac{\mathbf{q}(\mathbf{q} \mathbf{H})}{q^{0}+m}+\frac{\mathbf{q}}{2}\left(f^{0}-\frac{(\mathbf{q} \mathbf{f})}{q^{0}+m}\right)\right]-\frac{\mathbf{f}}{2} .
\end{aligned}
$$

Thus, the neutrino spin precesses around the direction $\mathbf{B}_{0}$ with the frequency $\omega=$ $2 m\left|\mathbf{B}_{0}\right| / q^{0}=2 \pi|\mathbf{q}| /\left(q^{0} L_{\text {osc }}\right)$. It is not difficult to see that the spin vector direction corresponding to stationary states $\boldsymbol{\zeta}_{t p}$ is connected to the effective magnetic field as follows:

$$
\boldsymbol{\zeta}_{t p}=\frac{\mathbf{B}_{0}}{\left|\mathbf{B}_{0}\right|}=\frac{m L_{o s c}}{\pi|\mathbf{q}|} \mathbf{B}_{0}
$$

This fact explains the stationarity of states with $S_{0}^{\mu}=S_{t p}^{\mu}$.

If neutrino possesses the fixed helicity in the initial state, i.e.

$$
S_{0}^{\mu}=S_{s p}^{\mu}=\frac{1}{m}\left\{|\mathbf{q}|, q^{0} \mathbf{q} /|\mathbf{q}|\right\}, \quad \boldsymbol{\zeta}_{0}=\boldsymbol{\zeta}_{s p}=\frac{\mathbf{q}}{|\mathbf{q}|},
$$


then formula (42) simplifies to the result widely discussed in the literature, which was mentioned in Introduction.

Clearly, our arguments based on the consideration of vector and axial currents and the final formula (42) are illustrations for neutrino propagation through background media. As it is commonly known [30, in a relativistic case only the integrals of motion are well defined observables.

\section{Conclusions}

In this way we obtained the exact solutions of the Dirac-Pauli equation for neutrino in dense matter and electromagnetic field. It was demonstrated that if the neutrino production occurs in the presence of an external field and a dense matter, then its spin orientation is characterized by the vector $S_{t p}^{\mu}$ instead of the vector $S_{s p}^{\mu}$. Using both the stationary (30) and the nonstationary (27) solutions it is possible to calculate the probabilities of various processes with neutrino in the framework of the Furry picture. Due to the time-energy uncertainty relation the considered states of neutrino can be generated only when the linear size of the area occupied by the electromagnetic field and the matter is comparable with the process formation length. This length is of the order of the oscillations length.

\section{Acknowledgments}

The authors are grateful to A. V. Borisov, O. F. Dorofeev and V. Ch. Zhukovsky for helpful discussions. This work was supported in part by the grant of President of Russian Federation for leading scientific schools (Grant SS - 5332.2006.2).

\section{References}

[1] Fukuda Y et al. (Super-Kamiokande Collaboration) 1998 Phys. Rev. Lett. $\mathbf{8 1} 1562$ (hep-ex/9807003)

Ahmad Q R et al. (SNO Collaboration) 2001 Phys. Rev. Lett. 87071301 (nucl-ex/0106015)

Eguchi K et al. (KamLAND Collaboration) 2003 Phys. Rev. Lett. 90021802 (hep-ex/0212021))

Ahn M H et al. (K2K Collaboration) 2003 Phys. Rev. Lett. 90041801 (hep-ex/0212007)

[2] Pontecorvo B 1957 Zh. Eksp. Teor. Fiz. 33549

Pontecorvo B 1958 Sov. Phys. JETP 6429 (Engl. Transl.)

Pontecorvo B 1958 Zh. Eksp. Teor. Fiz. 34247

Pontecorvo B 1958 Sov. Phys. JETP 7172 (Engl. Transl.)

[3] Maki Z, Nakagawa M and Sakata S 1962 Prog. Theor. Phys. 28870

[4] Bilenky M, Giunti C, Grifols J A and Massó E 2003 Phys. Rept. 37969 (hep-ph/0211462)

Giunti C 2007 J. Phys. G: Nucl. Part. 34 R93 (hep-ph/0608070)

[5] Mohapatra R N and Pal P B 2004 Massive Neutrinos in Physics and Astrophysics (Singapore: World Scientific)

[6] Lee B W and Shrock R E 1977 Phys. Rev. D 161444

[7] Fujikawa K and Shrock R E 1980 Phys. Rev. Lett. 45963

[8] Schechter J and Valle J W F 1981 Phys. Rev. D 241883 
[9] Voloshin M B, Vysotsky M I and Okun L B 1986 Zh. Eksp. Teor. Fiz. 91754

Voloshin M B, Vysotsky M I and Okun L B 1986 Sov. Phys. JETP 64446 (Engl. Transl.)

[10] Lim C -S and Marciano W J 1988 Phys. Rev. D 371368

[11] Akhmedov E Kh 1988 Phys. Lett. B 21364

[12] Borisov A V, Ternov A I and Zhukovsky V Ch 1988 Izv. Vyssh. Uchebn. Zaved. Fiz. 31 (No 3 ) 64 Borisov A V, Ternov A I and Zhukovsky V Ch 1988 Sov. Phys. J. 31228 (Engl. Transl.)

[13] Smirnov A Yu 1991 Phys. Lett. B 260161

[14] Akhmedov E Kh, Petcov S T and Smirnov A Yu 1993 Phys. Rev. D 482167

[15] Nunokawa H, Semikoz V B, Smirnov A Yu and Valle J W F 1997 Nucl. Phys. B 50117 (hep-ph/9701420)

[16] Dvornikov M 2007 arXiv:0708.2328 [hep-ph]

[17] Wolfenstein L 1978 Phys. Rev. D 172369

[18] Mikheyev S P and Smirnov A Yu 1985 Yad. Fiz. 421441

Mikheyev S P and Smirnov A Yu 1985 Sov. J. Nucl. Phys. 42913 (Engl. Transl.)

[19] Pal P B and Pham T N 1989 Phys. Rev. D 40259

[20] Nieves J F 1989 Phys. Rev. D 40866

[21] Nötzold D and Raffelt G 1988 Nucl. Phys. B 307924

[22] Pantaleone J 1991 Phys. Lett. B 268227

[23] Foldy L L 1952 Phys. Rev. 87688

[24] Bargmann V, Michel L and Telegdi V L 1959 Phys. Rev. Lett. 2435

[25] Lobanov A E and Studenikin A I 2001 Phys. Lett. B 51594 (hep-ph/0106101)

[26] Lobanov A E and Pavlova O S 1999 Vestn. MGU. Fiz. Astron. 40 (No 4$) 3$

Lobanov A E and Pavlova O S 1999 Mosc. Univ. Phys. Bull. 54 (No 4) 1 (Engl. Transl.)

[27] Lobanov A E 2006 J. Phys. A: Math. Gen. 397517 (hep-ph/0311021)

[28] Lobanov A E 2005 Phys. Lett. B 619136 (hep-ph/0506007)

[29] Zhukovsky V Ch, Lobanov A E and Murchikova E M 2006 Phys. Rev. D 73065016 (hep-ph/0510391)

[30] Landau L D and Peierls R 1931 Zs. f. Phys. 6956 